\title{
Investigating Media Use and the Television User Experience in the Home
}

\author{
Emmanuel Tsekleves, Roger Whitham, Koko Kondo and Annette Hill \\ Brunel University, Kingston Lane, Uxbridge, UB8 3UE, UK \\ Lancaster University, Lancaster, LA1 4YW, UK \\ University of Westminster, Northwick Park, Harrow, HA1 3TP, UK
}

emmanuel.tsekleves@brunel.ac.uk, r.whitham@lancaster.ac.uk, k.kondo@wmin.ac.uk a.hill@wmin.ac.uk

\begin{abstract}
In this paper we report on a study conducted in 2007 and 2008 looking at the media use habits of 27 families in the Greater London area. The project builds on previous work studying media use within a similar group in 2006. The study investigated attitudes towards different types of media and the role Television (TV) currently plays and could play within the home environment. To facilitate the study we rapidly prototyped an experimental home media device and asked participants to use and respond to it. We explored issues of interactional simplicity and sharing media using a TV and employed the experimental device as a focal point for discussion and the generation of new ideas. Our key findings indicate a strong desire for services which support media presentation and consumption through the TV (combined with a suitable control device) and cater for social interaction within the home such as sharing photos and videos with other household members. In addition we found a strong user preference for services that offer fast and immediate access to specialised online activities, such as quick checks of e-mail accounts and social networking services.
\end{abstract}

\section{Keywords}

Interactive Television, user studies, media use in the home, sharing media, experimental device, user experience.

\section{INTRODUCTION}

Media consumption within the home has seen substantial change in recent years. New forms of media and associated devices have become prevalent in domestic settings, altering the types of entertainment and information-centric activities individuals engage in. With increasingly general forms of media [1], such as the World Wide Web, come increasingly complex systems and the risk of an accompanying increase in the difficultly of using them.

Our research into media use in the home, along with that of others [1, 2, 3], shows that this increase in media availability and complexity has substantially different effects on different groups of users. In an exemplary and frequently documented trend, older individuals are typically less interested in, and able to interact with, the latest media devices and services.

This paper documents ethnographic research looking at the interaction of diverse media in the home and the equally diverse needs of the individuals who engage with them. Comparisons are made both between and within co-residential groups. Our research is focused around television (TV) as a common denominator of entertainment and information access within the home in the UK. A family TV often offers a high quality audio and visual experience compared with other devices in the home. It is also typically situated prominently in a shared social space, such as the living room or kitchen. These common features of TV usage, combined with the simplicity of the core TV user experience (a power button, channel and volume controls) make for a device all members of the family can understand, situated in an environment suitable for shared consumption of media.

After considering related literature, this paper describes our study in two distinct parts. Firstly we describe the process of engaging with study participants and finding out about their current and ideal media use. After reporting on these results we then describe how these findings drove the design of an experimental second screen device then presented to study participants to help gather further information on their attitudes and preferences towards media use. 


\section{PROJECT BACKGROUND}

BITe (Barriers to Interactive Television) is funded by the Media and Moving Image component of WestFocus ${ }^{1}$, a UK Government funded (HIEF) consortia of universities in the west of London working together to promote knowledge transfer between academia and industry. It involves collaboration between Brunel University and the University of Westminster. The overall aim of the project is to develop knowledge of the domestic digital media environment, including interactive Television (iTV) and suggest possible solutions based on audience/user perspectives. A combination of methods were used when engaging with participants, including interviews, observation and creative tasks. All households involved in the study were connected to both digital TV and broadband services and located in the Greater London area.

The project involved two phases. The first phase (2006-2007) investigated digital entertainment technologies in the home, exploring barriers to iTV in a household environment. This involved user reactions (likes, dislikes, frustrations, preferences) and technological issues and experiences associated with iTV services. It addressed several of these by developing a novel 'second screen' prototype remote control device in response to these findings and tested it with participants in their own homes. The project used a combination of methods involving interviews and observations with 20 households in the London area. For further information on this first phase of the project please see [19].

The second phase of the project (2007-2008) followed the same basic structure as the first phase, probing further into the use of digital entertainment technologies at home, exploring barriers to digital media engagement and the personalisation of digital media services and devices. A series of ethnographic studies were conducted involving repeated visits to 27 Greater London households with digital TV and broadband, leading in the latter phases to the development of an experimental device to facilitate further exploration of attitudes, expectations and barriers surrounding iTV and digital media use in the home. This paper reports on this second phase of the project.

\section{RELATED WORK}

\subsection{Media Use in the Home}

Co-resident groups of individuals are likely to make use of shared sources of information and entertainment. The home environment may contain all manner of information devices ranging from a wide-screen TV in the living room to individual laptops or mobile phones spread throughout the home. All such devices play complex social roles within the group as well as fulfilling objective information-access functions. Understanding the social context of media use in the home plays a vital role in informing the design of information devices and media forms, yet this topic is frequently overlooked [4]. The literature suggests that there is still a lack of studies on the context of media use in the home, in addition to a lack of empirical data on users' media consumption in a domestic setting [5].

Gaunlett's and Hill's is one of the most relevant and comprehensive media studies conducted in a domestic environment. It involved 500 UK participants over the course of 3 years and investigated people's TV use in a domestic setting [6]. Some key findings include the realisation that TV is a locus of attention and social interaction. It is prominently placed in the house and forms a central part of the family's social life with other interactions taking place around it and through the watching of it. Also viewing habits (different genres of TV show and times people watch TV) differ immensely between different age groups, genders and with personal interests. Other media devices such as the video recorder seemed to be integrated well into the TV

environment and welcomed by most viewers. Gaunlett and Hill found also that TV programming can be divided into three types: favourite programmes, which would always be watched or recorded, non-favourite programmes, which would be watched routinely and other programmes which would be watched because they happened to be on and seemed to be interesting [6].

Several other media studies conducted in a domestic environment evaluate a particular media technology [7, 8, 9]. These studies are centred solely on investigating user experience (on network digital devices, specific Internet Protocol Television prototypes and set-top boxes respectively) or investigate how people use multiple devices in various domestic and professional contexts, particularly looking at the interactions and information exchange between and across the devices [10], instead of focusing on media use and user experiences with media.

The media use studies most closely related to ours are those conducted by Obrist et al. [5] and Bernhaupt et al. [11]. These studies investigate media consumption with TV as the focal point. Both papers report on ethnographic studies that explore user's requirements and experiences of interactive TV within Austrian home environments, with 16 and 12 households participating in the two studies respectively. Another user study explores the use of TV and new media in the home environment [12]. This study, however, focuses solely on media consumption of younger members of the household such as children and teenagers, overlooking adult and elder household members' media use. Another study by Seager et al. investigated the use of 'triple-play' services (TV, Internet, Personal Computers (PCs)) in the home [1]. They conducted a study involving 40 households in the UK and France, conducting most interviews outside of the home itself with the use of self-documented pictures of household technologies. The majority of their study considered the different devices employed in the home. Some of their key findings involved the concurrent use of PCs and TV in the same room, especially in terms of performing quick checks of email and brief

\footnotetext{
${ }^{1}$ See: http://www.westfocus.org.uk .
} 
access to online information sources while watching TV. Seager et al. also documented user preferences for multiple display panels, user frustrations with DRM systems and incompatibility of devices and formats. Simons also reports on a media study on the impact of digitisation on existing Television practices [13]. This was conducted in May 2008 in Flanders in Belgium and included 80 respondents from various age groups. The main findings of this study suggest that viewers adjust the TV programme around their daily routines and in order to suit their own needs, thus making extensive use of time-shifting technology. In addition to this the participants of this study did not show an interest in interacting with TV programmes to control the programme content. Another study regarding the use of media and their integration with TV has been also conducted in Belgium involving 15 participants [14]. According to this study, watching TV has priority over playing gaming consoles on the home's main/shared TV set. Moreover, participants make a distinction between the use of different media and in particular in using the PC for going online and the TV for broadcasting and entertainment. Finally this study found that the use of the different media devices in the household complement each other instead of competing one another.

\subsection{The TV User Experience}

TV is often seen as being at the epicentre of the household media consumption [14]. It is principally seen as an entertainment activity and part of leisure experience in the domestic environment [15, 16, 11, 17]. Additionally, TV is regarded as a lean-back medium with its users often ascribing a relaxing and passive attitude towards it [18]. These notions of the TV user experience have, however, been altered by the advent of iTV in the home environment, allowing a traditionally passive medium to support a two-way interactive experience.

Several studies have shown that interactivity in the TV domain is not always welcomed by viewers, and in some cases, may be disruptive to the TV user experience $[15,16,19,20]$. This is especially likely as iTV services attempt to transform the TV from a lean-back to a lean-forward medium. The level of interactivity is not only limited by the potential of the technology, namely display mechanisms, hardware and interaction models [21, 22], but also by the user's willingness to interact [5].

The TV user experience benefits more from an optional interaction model, where the user decides how much interaction they wish to engage in, if any. As Chorianopoulos indicates [15]:

"On one hand, a fraction of the viewers impulsively selects a program to watch, there is also a need to support familiarity with a small set of channels. As a consequence, designers should assume that most TV viewing starts with familiar content, but it might continue with browsing of relevant items. As a principle, instead of information seeking, support relaxed exploration. Therefore, content navigation support should be subtle and not be enforced to the users."

Most of the media studies conducted on a domestic setting (see previous section) placed great emphasis on TV as the core media consumption and entertainment device in the home. TV has been the centre point of the entertainment experience with all or most household members making regular, if not always shared, use of it. It is interesting that although TV is usually controlled by a single person, it is clearly a medium that supports social activities in the domestic setting $[11,15,17]$. Geerts et al. have conducted extensive research on the subject of TV and sociability. They have published findings on TV programme genres that promote social interaction by watching TV together over distances by sending annotated video clips and messages to family or friends both synchronously and asynchronously [2]. Their study involved 36 participants in a simulated living room environment. The most interesting findings were that news and films are the most popular genres people talked about and that TV is the most popular device for viewing long and high quality video content. Harper et al. also found that even in the more personalised medium of mobile TV, watching TV on mobile phones appears to be a social activity too [3]. Furthermore younger members of the household, despite their frequent reliance on PCs and the Internet for entertainment purposes, also make use of the TV as or in support of social activities, though their parents often have first say in what is watched [12]. Williams et al [23] have also conducted research in this area, as part of the TA2 EU funded project. They involved 16 families across four European countries (Sweden, Netherlands, Germany and the UK) to study their social communications habits. Their findings indicate that games are considered by the majority of the participants as an important social activity and use of self-created photographs are employed as a means of stimulating and supporting social communication. Furthermore, Williams et al found that although most families did not make regular use of video communication (e.g. Skype), some of them appreciated the feeling of "presence" that an always-on video feed creates particularly in cases of an absent family member.

The TV is also often seen as comfortable and familiar. As such it can be perceived as a less intimidating technology to individuals less used to interacting with digital media, especially when compared to modern day PCs [12]. For elderly people the burden of accessing the Internet through the TV is lower than buying and learning to interact with a PC [5].

In the contemporary Western household environment there are diverse media devices (TV, Internet-enabled PC, MP3 players, digital cameras, etc.) and for each device there are multiple different content sources [15]. For example, through a typical TV one can find broadcast TV programmes, recorded programmes stored on the TV's Personal Video Recorder (PVR) and access to the Internet. This latest trend brings into the light the issue of increasing complexity of media access in the home and the creation a user experiences that are at odds with the simplicity of the TV user experience which predated these new features. 
In the first phase of the BITe project we found that study participants generally made limited use of the wide array of interactive features available [19]. Instead they concentrated on the most familiar and essential functions. The Electronic Programme Guide was a feature used by nearly all participants, while specialised interactive features unique to specific TV programmes or channels were rarely used. A recurring theme in the study's results was the desire for speed and simplicity with rapid and direct access to content. The interactive functions participants most favoured were those that facilitated rapid access to content with a minimum of fuss. The added complexity of multiple media sources and control mechanisms makes this requirement increasingly difficult to satisfy.

In this paper we use the term 'core TV user experience' to refer to a set of very basic interactions and content consumption patterns around the TV. The basic controls of analogue and digital TV alike, power on/off, volume up/down, channel selection, are easy to engage with and provide instant access to and movement between content. These controls provide instant, simple and now familiar access to high quality content. We feel this user experience is something with which almost all TV users can engage. Additional interactions beyond this may be possible, but should be optional if they do not compromise the core user experience itself.

Given the social aspect and nature of TV, its familiarity amongst the community of less experienced digital media users and its synthesis of different media sources, one could take the TV paradigm and experience to other media in the home (such as photos, video-clips, music and many more). This is one of the concepts of the ideas we most prominently explored during the study described in this paper.

\subsection{Use of Second Screen Devices in the TV Domain}

Although the focus of our research and this paper is not on the design and development of a portable second screen device, but rather on the concepts behind it, we still believe it appropriate to offer a brief section on this.

The concept of employing secondary screen devices in the TV environment is not a new one. In fact several researchers have designed and developed portable secondary screen systems for the control of TV content by more than one user simultaneously. These can be divided into two distinct categories, depending on the main motive behind the design. The first category includes secondary screen systems employed in the control of TV programmes and services. In 1996 Robertson et al. conducted a study employing a Personal Digital Assistant (PDA) in conjunction with a TV and set-top box to demonstrate an estate agency service [24]. Also Karanastasi et al. developed a prototype system that enables people to use mobile phones and PDAs to personalise and interact with a TV guide [25]. In their study Park et al. made use of sketch-based conceptual design to investigate the control of TV in a multi-user context with mobile phones [26]. Epelde et al also investigated the control of TV from a PDA [27]. However their motivation behind this was the creation and implementation of an accessible platform based on the Universal Remote Console Framework standard for the support of user interfaces for viewers with special needs, such as the visually impaired.

There are also studies that investigate the use of secondary screen devices beyond the control of TV. More precisely, commercial solutions such as MiXTV employ mobile messaging (both text-SMS and multimedia messaging-MMS) to interact with TV services, such as quizzes, game and voting and enrich the regular TV programming by displaying the messages and photos sent by viewers [28]. Cesar et al. have also contributed considerably in this area by developing a secondary system that enables viewers to enrich and author TV programme content [29] as well as share it with family and friends [30]. The introduction of a secondary screen in the TV domain has several advantages. Some of them are summarised below:

- It offers the opportunity to remove the need to show graphical navigation elements on the main Television screen. This allows viewers to continue enjoying the broadcast experience, even when the TV guide or an interactive service is viewed by another person in the household, by displaying the graphics and programme info on the secondary screen. This has been explored by Cesar et al [31] and Cruickshank et al [19] who developed a prototype secondary screen system explicitly separating the viewing and interacting content, allowing viewers to select and view content without interrupting what was played on the main screen.

- It also offers the opportunity to extend the model of TV operation by offering a richer interaction mechanism than conventional remote control devices that as Cesar et al [32] have demonstrated can empower the viewer to manipulate and enrich as well as share the media content.

- It allows for the dynamic reconfiguration of the user interface to reflect a user's personal needs and desires and can be used to allow for personalisation of both graphical elements as well as personalisation of media and TV content. As Epelde et al [27] have demonstrated in their research, a secondary screen device with reconfigurable and pluggable user interface has the potential to respond to the personal needs of viewers, particularly users with impairments, such as visual and cognitive impairments.

\section{STUDY METHODOLOGY}

27 households in the Greater London area, each with access to digital TV and broadband, participated in the primary study on which this paper reports. This provided a total of 70 people aged $8-50+$, containing a balance of gender and socio-economic 
status (ABC1, C2DE - ABC1 refers to participant profiles of low to low-middle class income and C2DE to participant profiles of middle to upper-middle socio-economic status) [33]. Table 1 provides an overview of the selected participants in the study.

The study involved a total of four visits to participants' homes by researchers. The first visit took place between February and August 2007. This included 10 households who had participated in the first phase of the project in 2006 along with 17 new households. The aim of the first visit was to establish trust between the participants and the researchers and to gather information about general media use in the households. A second visit took place between August to October 2007. It included all 27 households and aimed at gathering information on participants' likes and dislikes, preferences and experiences with respect to media in the home. A third visit was carried out between November 2007 to January 2008. This visit aimed at collecting information on participant's use of media devices, media content, media device personalisation and multiplatform media.

Table 1. Overview of participating people in the study

A final, fourth visit was carried out between February to March 2008. The aim of the last visit was to investigate and evaluate the concepts of interactional simplicity, customisation, privacy and security. This was facilitated with the use of an experimental home media device, developed as a response to participant observations, feedback and comments from previous visits. The device acted as a vehicle for further discussion and generation of new ideas. The ethnographic observations made during the four visits to participant's homes were augmented with three multiple-choice questionnaires (visit 1: general media use, visit 3: use of media devices, media content, media device customisation, visit 4: privacy and security, media customisation, media interaction and sharing). Audio from 108 interviews was recorded for later analysis.

In this paper we first discuss the outcomes of the first three visits (section 5). We then turn to the design of an experimental home media device used in the fourth and final visit, describing how the outcomes of the first three visits informed the design of the device, and the responses of participants to the experimental device in the final visit (section 6).

\section{INITIAL RESULTS}

Exploring the role of the TV in the domestic context formed our key research priority. The feedback gathered from the participant responses informed us about user needs and directed our response in the creation of an experimental device. This paper does not provide sufficient space to report on all of the ideas we explored with participants. As such we report on some of the more interesting areas of dialogue that arose during the study.

\subsection{The Core User Experience of TV}

Studies from the project share similar perspectives to those of the literature (see 3.2) as well as contributing new knowledge on the subject. Our initial results indicated what is well established already in the literature $[5,6,11,14]$ showing that TV is associated with relaxation and the safety and comfort of one's home:

"...to watch TV is you must be comfortable and basically be at home" (Andrew)

TV is also closely associated with familiarity and ease of use for all age groups and especially for older adults. It forms a piece of technology viewers trust and know well in contrast to interactive Television, as the following participants emphasise:

"It comes back to the fact, we are certain age, and for us, TV is familiar, so we like watching Television, but don't play around with interactive Television" (Linda and Tom, middle-aged couple)

Furthermore, the instant access to content with the simplicity of the TV interaction model renders it a key entertainment medium in the home. This is also well established in the literature with several studies $[15,16,17]$ reporting similar views to the following participant responses:

"because the good thing with this is everyone can use it even young kids, just look at it, you are already on it, you don't have to show them, that's how easy it is" (Leon)

"for someone like me that is not into the computer in any sense of the words the telly is simplifying everything for me" (Kate)

Similar to Saeger's study results [1] we also found that the role of the TV is often contrasted with other media related activities in the household which require a more active user role, such as using a PC:

"TV is more like when I have bad day, and it's more like relaxation, internet requires you [to take] more actions. You have to engage with it”. (Lynn)

Apart from the above initial results that complement the literature our study also provided a set of novel results. In particular, it was interesting that the same views on the differences between the role of the TV and PC are shared among most members of the household, despite their age and differing requirements (e.g. interests). This is exemplified in a family discussion regarding the different experiences of using the Internet and watching TV: 
14 year-old daughter: “...because I think with internet, coz you need to absorb information as such, whereas with TV so much visual television, you can tell what's going on without listening such, or, so definitely I say internet requires more concentration whereas television is more relaxing. If I am ill or something, I wouldn't go on the Internet. I would sit and watch telly.”

Mother: "That's true. With Internet, you are more, you are involved, doing with a mouse, aren't you? Keyboard as well. Whereas [with TV] you just probably watch a programme for half hour or hour and Internet you are doing other thing."

Father: "You can't fall asleep on the internet, can you?"

In addition to the limited choice of options of the TV, we found that the instant visual feedback TV provides seems to also form a core part of the TV user experience that further contributes to ease of use:

"I think TV is a good idea cause, it doesn't seem to be complicated and the choice is really easy to make and you have a visual, you know, impact or knowledge of what is going on.” (Mike)

This is a function that users do not always experience with other media devices, such as the PC and the Internet, where visual feedback is not reliably simple and instant. For instance, moving between channels on a TV is instant and abrupt, compared with the inconsistent feedback and speed with which web sites respond to navigational input.

The universal access and simplicity of using the TV has a positive impact on how people perceive it. The cognitive cost of interacting with the underlying technology is fairly low, while at the same time TV offers high quality content. Interaction costs are low, especially when compared to a PC. In this context a highly appreciated feature of the TV medium is its optional interaction model. This enables the viewer to decide how much (s)he wishes to interact with the TV content and any associated services, such as Teletext and interactive applications. The following quotes are typical of viewer experiences with a set of features they can interact with, but only if they wish to and at their own convenience:

"I know, it's going further [interactive TV], if you are interested in something, and you can go further to find out more information, but I don't use it so often.” (Diana)

“I don't really use them [interactive TV services]. I think the player camera became better, I don't know; and different commentators and things. But normally when I am watching football, I just watch football I don't really care if it is interactive or not." (Andy)

It was observed that participants tended to prefer interactive features which help them make decisions on what to consume and require minimal effort. This is especially true with optional interactive functions, such as the 'information function', which offer the viewer more information about programmes, when and if needed:

"we use it [information function] a lot. Lots of shows, Simpsons or Star Trek, I have seen them all, and then I press ' $i$ ' to see which episode it is, and I can decide if I watch it or not." (Kathrin)

\subsection{TV as a Shared Resource}

In addition to the association of relaxant with the TV, participants also associated social activities with the medium. For them TV is a shared experience. It usually takes a prominent place in the social space of the living room. It enables a common entertainment activity within the domestic environment, which encourages social interaction among household members during the content viewing experience and afterwards, as several studies have reported $[2,11,15,17]$. It should be noted here, that in this as well as the following sections, the word "share" refers to sharing of media through co-consumption from the same device, which Harper et al refer to as new forms of shared, co-proximate experiences [3].

"It is a family thing isn't it. You can interrupt because you can talk about what's going up and down and everything, which is funnier isn't it. Come on burn it down or something like that” (Daisy)

Watching TV is regarded as a more sociable activity that allows sharing of media among the household members and particularly among family members, as a mother explains and William's et al also indicate in their findings in this topic [23].

"linking the computer with the TV is a good idea cause it would make it possible for Andrew to share her stuff with all of us, so it open's it up a bit, it's more communicative" (Connie)

An interesting and repeating theme in our study was the social aspect of TV use. This was especially emphasised when participants contrasted TV use with PC and Internet activities. The PC was seen by participants as a solitary activity, which by its nature discourages social interaction and media sharing in the household. This was indicated by several participants expressing a desire for a way of sharing media content stored on the PC and Internet with all family members on the TV screen.

“[on the PC] it's quite difficult, back stretching to see over his shoulder it's better to share it over the TV” (Kate)

“...she does drama and singing and they've got a MySpace page and every now and then you might get a clip or Kate on the computer singing. And how many evening have we been sitting down here and heard her laughing and we've all had to run upstairs in that little room and have a look" (Katie) 


\subsection{TV and Multi-platform Media}

Today, several devices such as mobile phones, portable game consoles (e.g. Nintendo DS), pocket PCs can perform multiple media-related tasks. Several participants expressed a positive view on multi-platform media. For adults having different media sources is regarded as very good, including old media such as magazines and radio especially for news and information gathering, as well as catching up on missed TV programmes. This is in agreement with studies $[4,7,14]$ conducted in this area, which report results consistent with responses from the participants in our study.

Mother: "I just recently downloaded iPlayer BBC iPlayer, because I missed something."

Daughter: “and if you miss the something on channel four, you can watch on channel four website."

Table 2. Simultaneous use of media devices in the home

Teenagers and children tend to use multi-platform media mostly for playing games from relevant websites, such as the Cartoon network or Disney channel, where they can relate with the characters in the games.

Figure 1. The Experimental device: TV as a portal to other media.

Despite the positive view on multi-platform media, users do not often access a wide range of media from portable media devices, such as accessing the Internet from their mobile phones, and other portable media devices. This is due to device complexity and the limited number of websites that are being specifically designed to run on a mobile device (scaling, design and performance).

When asked whether they use multiple devices at the same time most participants responded positively with 86\% saying they perform multiple media-related tasks simultaneously, such as phoning and watching TV, using a PC and watching TV at the same time and several more. Table 2 indicates that TV accounts for four out of the five most popular simultaneous media device uses, with $64 \%$ of the respondents watching TV while performing other media-related tasks.

In this study we also explored the use of other non-TV-related media such as photos and videos, particularly in the context of the PC use and the Internet. In a series of questionnaires we found that $86 \%$ of participant households view photos on the Internet and $78 \%$ of them also watch video clips online. Although nearly eight out of ten households consume photos and videos online, only half of them share or upload photos on the Internet and even fewer, approximately three in every ten households, upload or share video clips online.

Following the social and shared aspect of the TV user experience, we explored the use of the TV in consuming other media. Our study indicated a new tendency of households to share some media content through their TV set. More technical savvy households connect their digital cameras to share their pictures with other family members as well as friends. This allows them to showcase to family members and friends their own experiences, such as family events, celebrations and holiday as well as use it as a means of sparking discussions.

"that's what we tend to do, don't we, cause we've been doing it straight off your camera onto the TV and looking at them with friends which is quite fun to do" (Jane)

"I like the idea of being able to see them on the telly, it's nice when you come back from holidays you sit down and all look at the photos together, especially if you had a really good party and there is some funny photos of everybody getting all round together" (Kate)

\section{THE EXPERIMENTAL DEVICE}

For the fourth and final visit to study participant's homes we developed an experimental home media device. The device functioned somewhat like a "technical probe”, as described by Hutchinson et al [34], but crucially was only given to participants for the length of a single visit (30-60 minutes). The device was not the subject of a user interface or usability evaluation of any kind during this study, instead it was used as a focus for the final visit, responding to and making concrete many of the ideas discussed with participants in the previous visits and allowing the participants to imagine how similar, fully-functional devices might function in the future. 


\subsection{Our Design Response}

Our design response was strongly influenced by study participant's views on the core TV user experience, gathered during the first three visits of our study and in the previous phase of the project. Our aim with the experimental device design was to ensure the maintenance of the core TV user experience (instant access, simplicity, familiarity, low interaction cost, high quality, optional access to additional content) while also presenting some novel content sharing ideas for discussion with study participants.

The very positive response of households to sharing media on the TV, preferences for accessing multiple media types from a single device and the use of several media-related devices at the same time created new opportunities for media convergence.

This led us in the design and development of a second screen experimental device, aimed at making interaction more intuitive and less intrusive, especially for those individuals who see TV solely as a relaxing activity. The resultant experimental device was intended to realise the following ideas:

- $\quad$ The core TV user experience as a counterpoint to modern digital media experiences.

- $\quad$ TV as a social activity: the shared experience of the TV is ideal for other non-TV-related media sharing, such as photos, videos and music

- $\quad$ TV as a common denominator: all members of the family can understand TV content and engage in basic interaction with the technology.

Our experimental touch screen device enabled the TV to function as a portal to access and share different types of media. This included the viewing and sharing of photos and videos from YouTube and other online sources. Some features were implemented to be fully functional, while others were included simply a non-interactive user-interface visualisations on the device itself. For example, the Electronic Programme Guide (EPG) used real-time programme data and was fully interactive, but in contrast selecting a YouTube video did nothing at all. We made sure to point out these limitations in functionality to the study participants and asked them in all cases to focus on how they would want such a device to function, rather than the specific design features of the experimental device with which they were presented. We do not include a detailed discussion of the design of the device as this was not evaluated in our study, but in outline the device simulated the following features:

- $\quad$ Electronic Programme Guide - A grid showing TV channels and programmes. Further information on programmes could be access in a popup window, also allowing reminders for programmes to be set and programmes recorded.

- $\quad$ Favourite channels list - Always available at the bottom of the user interface (UI).

- $\quad$ User-profiles - Allowing rapid switching between different user and their personal settings.

- $\quad$ TV volume control

- $\quad$ TV Show list - Allowing a personalised list of favourite TV shows to be created. Selected shows would be highlighted in the EPG and newly recorded episodes flagged. Customisation of this list was achieved through drag and drop from a list of all TV shows available.

- $\quad$ Films list - A list of purchased film available for viewing. Additional films could be purchased using a Pay-as-you-go system.

- $\quad$ Photo gallery list - Preloaded photo galleries could be view on the device and mirrored on the TV if desired.

- $\quad$ Shared media list - Items from the internet such as YouTube videos could be viewed on the device and the TV.

Figure 2. Bringing different media to the TV.

In this study we were only interested in participant responses to the ideas and concepts behind the experimental device, not the device itself and user interface employed. Our main interest was in revealing households' opinions after making use of the experimental device and in generating discussion as to where this could lead or be extended to. The experimental device was used as a focal point and catalyst for discussion. As such we did not conduct an evaluation of the usability and user experience of the experimental device in this paper.

\subsection{The Device}

The experimental device used in this study was designed to provide a realistic media-use experience for study participants in their own homes. The device connected wirelessly to a laptop, which was in turn connected to participant's TVs. This allowed actions performed on the experimental device to be instantly reflected on the TV within participant's normal viewing environment. The 
device provided various simulated functions, allowing control over the TV channel showing and allowed participant's to display photos on the TV screen.

The second screen device itself was created using a Samsung Q1 Ultra running Microsoft Windows XP. The device’s dimensions were approximately $23 \times 12 \times 23 \mathrm{~cm}$, with a 7 inch screen. The user interface displayed on the device was implemented using Adobe Flash connected wirelessly to a Java application running on a separate laptop, also running Microsoft Windows XP.

The UI itself was controlled entirely with the touch screen. The buttons visible to the right and left of the device's screen in figures 1, 2, 3 and 4 were not used in the study. Most actions were performed by touching regions of the screen, for example touching channel logos to change channels, or TV programmes to view information about a specific show. Scrolling of lists and panes was performed with a dragging gesture.

\subsection{Participant Responses}

Using the experimental device as a focal point, we generated discussion on whether and how households would employ similar devices in sharing media between household members. The experimental device included a number of personalised photos and videos that the user could either view privately on the device itself or display to the main TV for others to see. Here we present some of the participant responses and suggestions we gathered.

Overall people showed positive attitudes towards accessing different media from the experimental device and projecting the contents to the TV. $96 \%$ of participants liked the idea of being able to access a range of media (TV, photos, video) and $91 \%$ expressed the wish to share photos and videos with family and friends using such a device.

“you could be in the same room and enjoy different media, that would be great, wouldn't it?” (Carole)

"I think if you gonna go for the full mouthy, that's probably what the future means to everybody, going forward and incorporating all different presentations of media, having everything all in one place seems to be the thing, doesn't it?” (Kelly)

When asked which type of media they would expect to share the most, participant responses varied depending on the device involved. If sharing media using the second screen device's screen, 43\% responded they would mostly share the photos, followed by TV shows with 25\%, $15 \%$ other videos (e.g. YouTube) and $12 \%$ films. Interestingly, in the case of media shared on the TV screen, participants responded that they expected to share TV shows most frequently (49\%), followed by photos (28\%), films (17\%) and then videos (6\%).

\subsubsection{Sharing Photos}

Most participants greatly appreciated the photo-sharing concept. Participants wanted to share photos with other family members, friends and even party guests.

"the photos is good and to be able to share it and very easily... with this I think it will cut the process of doing the complicated stuff, you know, of transferring, connecting, etc... [with this] you will not lose a lot of time” (Mark)

'I'd have put it in a photo album I'd have prepared it and it's great for when friends come round the same with you tube to watch clips which are funny as part as, you know, a party a conversation" (Garry)

Seager et al. also found in their study that users favoured services which make use of the social nature of the space, such as photo sharing, especially with family and friends [1].

\section{Figure 3. Browsing photos on the experimental device.}

Another participant suggested that the idea behind the experimental device enables an activity which was lost when domestic photo technologies became primarily digital, making possible again activities surrounding sharing pictures with other people.

"I like the photo idea, cause I always thought it was very sad when everybody when to digital cameras, whereas in the old days you had a film developed and you had something to look at, print and you don't tend with the digital camera to look at your pictures as often as you would if you had them in an album or whatever, but if you have them on here it would be quite easy to get to them it would make it a lot more accessible" (Jacky)

One participant saw a conceptual distinction between the situations and tasks of sharing photos and viewing them individually, highlighting the benefit of having a socialising activity whilst maintaining privacy.

“you come back from holiday where you've took a lot of pictures and you do a slideshow and invite friends then you project them on the TV...here is more like when I am by myself and I am like let me see my memories from holiday." (Carol) 
Figure 4. Projecting photos from the experimental device to the TV.

In this context, one young participant described how the experimental device could support privacy when watching video media too.

"say if you've got something personal like a video rather than it just coming up on the TV straightaway you can skip it, you don't give anyone a chance to see it, that's for you on private use and you just touch something else and you see something else on the screen, yeah that is a good idea" (Trevor)

An interesting observation was that the photo sharing idea appealed mostly to adults, parents and young children. Younger participants such as teenagers did not show much interest in sharing photos with their parents and family. For them this is more of a private activity which they would mainly undertake with a few friends and peers around a PC. For some younger participants the sharing of photos in the experimental device was seen as making the viewing of photos easier for their parents.

\subsubsection{Sharing Videos}

The idea of sharing of videos either streamed or downloaded from the Internet by projecting them on the TV appealed mostly to younger adults and especially teenagers. Parents preferred the idea of sharing photos to that of sharing online video clips.

"YouTube, yeah if you'd just found a funny clip and wanted to show the family. It'll be like oh look at this I show that today just bang that out, click on it and you can just show to everyone and have few giggles maybe" (Simon - 27 years)

"it would be to see videos on here that you would upload, then you would end up with your own private YouTube" (Jeff - 14 years)

One participant expressed the desire to expand the range of online video sources available on the experimental device to cater for a wider range of video content.

"I'd probably use the video if it was somewhere you could access stuff like people on Facebook, if you got access to that sort of stuff and you can see peoples' videos, like YouTube I guess that would be interesting” (Trevor - 16 years)

\subsubsection{Further Participant Suggestions}

The discussion generated from the use of the experimental device lead to several further suggestions from participants. Generally households responded very positively to the concept of accessing similar media to those available with the experimental device. When asked directly whether they would want similar access to media on their existing TV, 80\% responded 'yes', 8\% responded 'no' and $12 \%$ responded 'don't know'. Most study participants suggested the expansion of the media-sharing concept to incorporate music and Internet-related media.

Participants indicated that the social activities supported by the experimental device could also involve music. This idea was popular among younger age groups and was mainly expressed as a new means of sharing music content with people outside of the household, such as friends.

"like your iPod, it could be incorporated into it so you've got all your favourite music in there, then you play it on the telly instead of your iPod...oh yeah that would be cool" (Roger - 15 years)

"you could create your own playlist and when you have your friends around instead of switching the channels to find something you like you just have it on there and just play all of them” (Ann - 16 years)

A new theme that emerged from the discussion was that of 'quick Internet checks'. Several participants expressed the desire of having Internet access on the experimental device in addition to their own PCs. According to respondents, Internet access from the experimental device should be a simple way of enabling them to perform short web-related activities, such as finding favourite videos over YouTube or accessing email and Facebook accounts.

"I use a for lots of Internet things and I'd rather use that if I could cause it's touch screen it just makes it so much easier, I've got a laptop and it's always clicking, touch would be a really good function, you know reading deleting e-mails and stuff, it would be much user friendly than using a mouse" (Susan)

"It depends on your Internet usage; to check your e-mails quickly this would be perfect even the You Tube...that's good because it's mild very low intense Internet usage you don't have to do a lot of typing. For me when I go on the Internet I'll be doing a lot of typing so my preference is to use the laptop, but for small scale stuff it would be pretty useful but definitely useful if it has the equivalent little buttons to go directly to or directly to hotmail or something like that so it's kind of controlled Internet usage." (Garry)

When households were asked whether they would use the experimental device to share media from the Internet with others the majority responded positively. $74 \%$ of participants suggested they would do so with their family in contrast to $83 \%$ who would prefer to do this with their friends. 
When asked, participants would often suggest that the more media-related devices they could remotely control with the experimental device the better. Participants particularly wanted to use the experimental device to gain access to other media devices such as DVD players, Music Stereo, iPod and camcorders. When we posed the question of whether this might compromise the ease of using the core functions, some participants showed concern while others didn't see this as an issue.

\section{CONCLUSIONS}

In this paper we presented the results of our ethnographic study on the use of different types of media and the role TV currently plays in modern households. Based on the participant feedback we developed an experimental device to investigate further the concept of the core TV user experience as a counterpoint to modern digital media experiences and to explore shared use of TV in the home. The experimental device proved useful as a means of presenting new concepts to participants, and more importantly in generating further discussion.

Themes emerging from our research include the continuing importance of the core TV user experience, negative feelings towards iTV services and the desirability of devices and services specialised for specific online activities such as email and social networking. We explored ideas surrounding shared consumption of media along with the issues of user experience personalisation and privacy this raises.

In line with our previous work, interactive features of iTV that went beyond channel selection and planning viewing saw only limited use with our study participants. Many study participants were accustomed to deferring interaction with some services to more technically-savvy, and typically younger, members of the household. This said, all participants were willing to engage with the TV on a basic level, behaviour we sort to explore further with our experimental device. Study participants who found it challenging to use PCs valued the relaxing aspects of the TV user experience. Participants who made extensive use of PCs and the Internet for social and entertainment activities still valued the TV as a low-effort medium offering a relatively high quality and often shared entertainment experience.

The burden of using PCs was something especially highlighted by participant responses to our experimental second screen device. With limited or no prompting participants expressed the desire to use the device to engage in Internet-related activities in addition to using it to control the TV. This desire was present in those participants with a laptop computer available in the same space as their TV, suggesting a link with the simplicity and speed of interaction the device offered rather than merely its availability as a portable Internet device.

With PCs becoming more prevalent in the home and as common sources of entertainment, the value of the TV as a shared entertainment source available to all household members is at risk of being ignored. We explored different ways in which a household TV could be used to share media between family members, providing study participants with the means to try out coconsuming indicative media types in their own home. The mechanisms we used to do this we unrefined and not subject to testing here, but the discussion generated suggested a real value in making it easy for households to access media often restricted to a PC by using their TV. The ways in which household members might use such a system in every day life would be a suitable topic for further investigation.

The desire to share social space while consuming media was a common theme of our study. This applied least to teenagers, but was strong with all other groups of participants. The social activities surrounding TV are dependant on household members seeking to engage in shared entertainment activities, in turn requiring content of mutual interest. With increasingly diverse and specialised media available in the home, appropriate means to bring together content is likely to be valued by household members who also value the social side of TV use. Making the TV relevant and useful to household members is something we intend to explore in the future, both in social terms and using experimental devices and participatory design methodologies.

\section{REFERENCES}

[1] W. Seager, H. Knoche, M. A. Sasse . TV-centricity - Requirements gathering for triple play services. In Interactive TV: A Shared Experience TICSP Adjunct Proceedings of EuroITV. 2007. Springer. 274-8.

[2] D. Geerts, P. Cesar, D. Bulterman. The implications of program genres for the design of social television systems. In Proceeding of the 1st international Conference on Designing interactive User Experiences for TV and Video. UXTV '08, 291. 2008. ACM Press. 71-80.

[3] R. Harper, T. Regan, M. Rouncefield. Taking hold of TV: learning from the literature. In Proceedings of the 18th Australia Conference on Computer-Human interaction: Design: Activities, Artefacts and Environments OZCHI '06, 206. 2006. ACM Press. 79-86.

[4] V. Haines, V. Mitchell, C. Cooper, M. Maguire. Probing user values in the home environment within: A technology driven Smart Home project. Personal and Ubiquitous Computing. 11 (5). (2007) 349-359.

[5] M. Obrist, R. Bernhaupt, M. Tscheligi. Interactive television for the home: An ethnographic study on users requirements and experiences. International Journal of Human-Computer Interaction. 24 (2). Taylor \& Francis. (2008) 174 - 196. 
[6] D. Gauntlett, A. Hill. TV Living: Television, Culture and Everyday Life. Routledge, London, 1999.

[7] S. Bly, B. Schilit, D. W. McDonald, B. Rosario, Y. Saint-Hilarie, Broken Expectations in the Digital Home. Ext. Abstracts CHI 2006, ACM Press. (2006) 568 - 573.

[8] D. Vyas, G. C. van der Veer. Rich Evaluations of Entertainment Experience: Bridging the Interpretational Gap. Proceedings of ECCE-13, 2006, ACM Press. 137-144.

[9] J. O’Brien, T. Rodden, M. Rouncefield, J. Hughes. At home with the technology: An in-home computing and ethnographic study of a set-top-box trial. ACM Trans. Computer-Human Interaction. 6 (3). ACM Press (1999) 282-308.

[10] D. Dearman, J. Pierce, J. "It's on my other computer!": Computing with Multiple Devices. Proceeding of the twenty-sixth annual SIGCHI conference on Human factors in computing systems. 2008. ACM Press. 767-776.

[11] R. Bernhaupt, M. Obrist, A. Weiss, E. Beck, E. Tscheligi. Trends in the living room and beyond: results from ethnographic studies using creative and playful probing. ACM Computer Entertainment. 6 (1). ACM Press (2008) 1-23.

[12] B. Svoen. Consumers, Participants, and Creators: Young People's Diverse Use of Television and New Media. ACM Computers in Entertainment. 5 (2). ACM Press (2007) 1-15.

[13] N. Simons. "Me TV": towards changing TV viewing practices?. In Proceedings of the Seventh European Conference on European interactive Television Conference EuroITV '09. 2009. ACM Press. 219-222

[14] K. Naessens, A. Jacobs. The place of TV and other media in common gaming practices: a fluent marriage?. In Proceedings of the Seventh European Conference on European interactive Television Conference. EuroITV '09. 2009. ACM Press. 211-214

[15] K. Chorianopoulos. User Interface Design Principles for Interactive Television Applications. International Journal of Human-Computer Interaction. 24 (6). Taylor \& Francis. (2008) 556 - 573.

[16] K. Chorianopoulos, D. Spinellis. User interface evaluation of interactive TV: a media studies perspective. Journal of Universal Access in the Information Society. 6 (2). Springer-Verlag (2006) 209-218.

[17] K. Keeling, L. A. Macaulay, P. J. McGoldrick. DiTV and e-commerce among disadvantaged community groups. Journal of Behaviour and Information Technology. 26 (6). Taylor \& Francis (2007) 545-560.

[18] M. Gawlinski. Interactive television production. Oxford, Focal Press, 2003.

[19] L. Cruickshank, E. Tsekleves, R. Whitham, A. Hill, K. Kondo. Making interactive TV easier to use: Interface design for a second screen approach. The Design Journal. 10 (3). Ashgate (2007) 41-53

[20] K. Baker P. Hulsen. Multi-service models for interactive television. IEEE International Conference on Consumer Electronics. 2003, IEEE. 88 - 89.

[21] E. England, A. Finney. Managing Multimedia: Project Management for Web and Convergent Media, Book 2: Technical Issues. London, Addison-Wesley, 2002.

[22] L. Eronen P. Vuorimaa. User Interfaces for Digital Television: a Navigator Case Study. Proceedings of conference on Advanced visual interfaces. 2000, ACM Press. 276-279.

[23] D. Williams, M. F. Ursu, P. Cesar, K. Bergström, L. Kegel, J. Meenowa. An emergent role for TV in social communication. In Proceedings of the Seventh European Conference on European interactive Television Conference. EuroITV '09. 2009. ACM Press. 19-28

[24] S. Robertson, C. Wharton, C. Ashworth, M. Franzke. 'Dual device user interface design: PDAs and interactive television'. Conference on Human Factors in Computing Systems CHI 1996, Proceedings of the SIGCHI conference on Human Factors in computing systems: common ground. 1996, ACM Press. 79-86.

[25] A. Karanastasi, F. G. Kazasis, S. Christodoulakis. A natural language model for managing TV-anytime information in mobile environments. Personal and Ubiquitous Computing. 9 (1). Springer-Verlag (2005) 262-272.

[26] J. Park, M. Blythe, A. Monk, D. Grayson 'Sharable digital TV: relating ethnography to design through un-useless product suggestions’. Conference on Human Factors in Computing Systems CHI 2006. 2006, ACM Press. 1199-1204.

[27] G. Epelde, E. Carrasco, G. Zimmermann, J. Bund, M. Dubielzig, J. Alexandersson. URC based accessible TV. In Proceedings of the Seventh European Conference on European interactive Television Conference. EuroITV '09. 2009. ACM Press. 111-114

[28] S. Miller. Taking on the masses with mobile messaging TV. Comput. Entertain. 3 (2) ACM Press (2005) 6-6.

[29] P. Cesar, D. Bulterman, A. Jansen. Usages of the Secondary Screen in an Interactive Television Environment: Control, Enrich, Share, and Transfer Television Content In Proceedings of EuroITV 2008. 2008. Springer-Verlag. 168-177

[30] P. Cesar, D. Bulterman, A. Jansen. Social sharing of television content: An architecture. In: Proceedings of the IEEE Symposium on Multimedia (Workshops), 2007. IEEE. 145-150 
[31] P. Cesar, D.C.A. Bulterman, J. Jansen, D. Geerts, H. Knoche, and W. Seager, "Fragment, Tag, Enrich, and Send: Enhancing the Social Sharing of Videos," ACM Transactions on Multimedia Computing, Communications, and Applications. 5(3). ACM. (2009). 11-20

[32] P. Cesar, D.C.A. Bulterman, and J. Jansen, "Leveraging the User Impact: An Architecture for Secondary Screens Usage in an Interactive Television Environment". Springer/ACM Multimedia Systems Journal. 15(3). Springerlink. (2009). $127-142$.

[33] R. Bocock. Consumption (Key Ideas). London: Routledge, 1993.

[34] H. Hutchinson, W. Mackay, B. Westerlund, B. Bederson, A. Druin, C. Plaisant, M. Beaudouin-Lafon, S. Conversy, H. Evans, H., Hansen, N. Roussel, and B. Eiderbäck, Technology probes: inspiring design for and with families. In Proceedings of the SIGCHI Conference on Human Factors in Computing Systems. CHI 2003. ACM. 17-24. 